\section{CLINICAL SIGNIFICANCE AND PROGNOSIS VALUE OF WNT SIGNALING PATHWAY IN CERVICAL CANCER}

M Farazestanian*. mashhad university of medical sciences, gyneacology , mashhad, Iran

10.1136/ijgc-2021-ESGO.40

Introduction/Background* A major controversy in sentinel node (SN) biopsy of endometrial cancer is the injection site of mapping material. We compared lymphatic drainage pathways of the uterine cervix and uterine body in the same patients by head-to-head comparison of intracervical radiotracer and fundal blue dye injections.

Methodology All patients with pathologically proven endometrial cancer were included. Each patient received 2 intracervical injections of Tc-phytate. At the time of laparotomy, the uterus was exposed, and each patient was injected with 2 aliquots of patent blue $\mathrm{V}(2 \mathrm{~mL}$ each) in the subserosal fundal midline locations. The anatomical locations of all hot, blue, or hot/blue SNs were recorded.

Result(s)* Overall, 45 patients entered the study. At least 1 SN could be identified in 75 of 90 hemipelves $(83.3 \%$ overall detection rate, $82.2 \%$ for radiotracer [intracervical] alone, and $81.1 \%$ for blue dye [fundal] alone). In 71 hemipelves, SNs were identified with both blue dye (fundal) and radiotracer (intracervical) injections. In 69 of these 71 hemipelves, at least 1 blue/hot SN could be identified ( $97.18 \%$ concordance rate). In 10 patients, para-aortic SNs were identified. All of these nodes were identified by fundal blue dye injection, and only 2 were hot.

Conclusion* Our study shows that lymphatic drainage to the pelvic area from the uterine corpus matches the lymphatic pathways from the cervix, and both intracervical and fundal injections of $\mathrm{SN}$ mapping materials go to the same pelvic SNs.

\section{A VARIATION OF LAPAROSCOPIC OVARIAN TRANSPOSITION: THE OVARIAN PEDICLE SUSPENSION (PS TECHNIQUE)}

P Stanciu*, M Oikonomou, M Padwick. West Hertfordshire Hospitals NHS Trust, Gynaecological Oncology, London, UK

\subsection{6/ijgc-2021-ESGO.41}

Introduction/Background* Laparoscopic Ovarian Transposition (OT) has already been proven to be a safe and effective procedure to preserve ovarian function in patients receiving pelvic radiotherapy for a variety of gynaecological malignancies. Different techniques have been described.

Aim This video demonstrates our PS technique for OT in a 32-year-old patient with stage 1B3 poorly differentiated squamous cell carcinoma of the cervix who subsequently underwent radical chemoradiation.

Methodology/Technique Laparoscopy was performed as usual, using a $10 \mathrm{~mm}$ umbilical optic port and four $5 \mathrm{~mm}$ ports placed in both iliac fossae and high in both flanks. Thorough inspection of the peritoneal cavity revealed no evidence of disseminated disease. Approximately $100 \mathrm{mls}$ of free blood was seen in the pelvis. Both ovaries were slightly enlarged and the right ovary had a ruptured haemorrhagic cyst. She had previously developed OHSS after ovarian stimulation and egg retrieval. The uterus was bulky and retroverted. Both tubes were normal. All the upper abdominal organs looked normal and there was no evidence of disease on the ovaries or peritoneal surfaces therefore we decided to proceed to bilateral ovarian transposition.

Result(s)* Bilateral retrograde salpingectomy was performed using a Harmonic scalpel and specimens were sent for histology. Both pelvic side walls were opened and both ureters were identified. Both utero-ovarian ligaments were transected along with $2 \mathrm{~cm}$ of round ligament on both sides and ovarian flaps were created. The ovarian flaps were mobilised and the infundibulopelvic ligaments were skeletonised. The para-colic gutters were incised approximately $10 \mathrm{~cm}$ above the pelvic brim and were tunnelled. Both ovarian flaps were pulled through and stapled outside the irradiation fields to prevent them from falling back into the pelvis following the procedure. Titanium staples were used for easy identification of ovaries on imaging. At the end of the procedure both ovarian pedicles were tension-free with good mobility and no risk of necrosis or torsion. There were no intraoperative complications and the patient experienced a good recovery.

Conclusion* We consider that the ovarian flap allows the ovaries to have a degree of natural movement, while at the same time preventing torsion and minimising ovarian damage associated with the use of transfixed stitches.

\section{BEVACIZUMAB IN CERVICAL CANCER - THE EXPERIENCE OF A COMPREHENSIVE CANCER CENTER IN NORTHERN PORTUGAL}

A Vaz-Ferreira*, S Monteiro, AR Lopes, M Abreu, M Ferreira, S Sousa, P Redondo, D Pereira, J Savva-Bordalo. Instituto Português Oncologia do Porto Francisco Gentil, EPE, Porto, Portugal

\subsection{6/ijgc-2021-ESG0.42}

Introduction/Background* recent studies show that addition of anti-VEGF monoclonal antibody bevacizumab to platinumbased chemotherapy (P-CT) in advanced cervical cancer (ACC) treatment improves overall survival with an acceptable safety profile. The objective of this study was to evaluate bevacizumab outcomes in ACC treatment in a comprehensive cancer center (CCC) in Portugal.

Methodology we retrospectively reviewed consecutive medical records of ACC patients (persistent, recurrent or metastatic), $\geq 18$ years-old who were eligible for bevacizumab concomitant with P-CT, between 2015 and 2020. Primary endpoint was overall response rate (ORR) and secondary endpoints mortality and safety, assessed according to Common Terminology Criteria for Adverse Event v4.0. Descriptive analysis of main demographic, clinical and treatment variables were performed.

Result(s)* we identified 12 ACC patients with median age of $54(28-72)$. Metastatic disease was present in half of patients $(n=6)$, persistent disease in $3(25.0 \%)$ and recurrent disease in $3(25.0 \%)$ patients. Eight patients $(66.7 \%)$ had previously received platinum-based chemoradiotherapy. Median number of cycles P-CT was $6(5-10)$ and bevacizumab was 13 (1 - 64). The ORR was $66.7 \%$. Three patients $(25 \%)$ had complete response, $5(41.7 \%)$ partial response, 3 (25\%) stable disease and $1(8.3 \%)$ disease progression. The incidence of grade $\geq 2$ hypertension was $25 \%$, grade $\geq 3$ thromboembolic events $8.3 \%$ and fistulas (gastrointestinal/genitourinary) $25 \%$. The median follow-up time was 15 months. Patients discontinued bevacizumab due to unacceptable toxicity $(n=4,36.4 \%)$ or disease progression $(n=5,45.4 \%)$. At the cut-off date, 7 